Meta

Journal des traducteurs

Translators' Journal

\title{
Évolution de la distribution dans les moteurs à combustion intermittente
}

Jacques Lethuillier

Volume 27, numéro 3, septembre 1982

URI : https://id.erudit.org/iderudit/003490ar

DOI : https://doi.org/10.7202/003490ar

Aller au sommaire du numéro

Éditeur(s)

Les Presses de l'Université de Montréal

ISSN

0026-0452 (imprimé)

1492-1421 (numérique)

Découvrir la revue

Citer cet article

Lethuillier, J. (1982). Évolution de la distribution dans les moteurs à

combustion intermittente. Meta, 27(3), 305-318.

https://doi.org/10.7202/003490ar d'utilisation que vous pouvez consulter en ligne.

https://apropos.erudit.org/fr/usagers/politique-dutilisation/ 


\section{ÉVOLUTION DE LA DISTRIBUTION DANS LES MOTEURS À COMBUSTION INTERMITTENTE}

$\mathrm{Au}$ sens large la distribution (timing) pour un moteur volumétrique (displacement-type engine) à combustion interne désigne la commande, par le moteur lui-même, de ses organes auxiliaires comme les soupapes (valve), l'allumeur (distributor), la pompe d'alimentation (fuel pump), la pompe à huile (oil pump), la pompe à eau (water pump), l'alternateur (alternator, a.c. generator) et le ventilateur $(f a n)$. Au sens restreint, il s'agit de l'ensemble des organes commandant l'admission (intake) des gaz dans le moteur puis leur échappement (exhaust), ou encore de la fonction réalisée par cet ensemble.

Sur les moteurs thermiques (heat engine) à combustion interne de type turbomachine (non-displacement type), puisque les phases d'évolution du fluide moteur se déroulent en continu à l'intérieur d'organes distincts communiquant entre eux, la distribution n'a pas sa raison d'être. Au contraire, elle est essentielle pour la réalisation d'un capsulisme avec les moteurs thermiques du type à combustion interne.

Son rôle est alors si important que l'on distingue les moteurs d'après la façon dont se trouve assurée la distribution ou d'après la disposition des principaux organes servant à réaliser cette fonction.

\section{LA DISTRIBUTION SANS SOUPAPE}

Quand il est fait appel à un autre moyen que la soupape (valve) pour l'obturation (covering) des orifices ou lumières d'admission (intake port) et d'échappement (exhaust port), on parle de distribution sans soupape (valveless timing). Pareille disposition caractérise certains moteurs deux temps (two-stroke engine) et se retrouve sur les moteurs rotatifs Wankel (Wankel rotary engine), mais ce n'est pas là un mode de distribution très répandu.

\section{LA DISTRIBUTION PAR SOUPAPES}

\section{La soupape}

Sur la grande majorité des moteurs thermiques modernes à pistons, la distribution met en jeu des soupapes, qui sont des obturateurs formés essen- 
tiellement d'une tête (head, disc, plug) se raccordant par une collerette (collar) à une tige (stem) que termine la queue (tip, valve stem end).

Les soupapes plates ou soupapes à sì̀ge plan (fat-type valve, flat valve, plate valve), dont la portée est une surface plane perpendiculaire à l'axe de la tige, ont fait place de nos jours à des soupapes à siège conique. La tête de ces dernières comporte une partie chanfreinée que l'on appelle collet (valve head face) et qui, à la fermeture, forme joint avec le siège de soupape (valve seat) lui servant d'appui.

Le siège de la soupape fait partie de la culasse (cylinder head) si le moteur est à soupapes en tête (overhead valve type, valve-in-head type) et du bloccylindres (cylinder-block) s'il s'agit d'un moteur à soupapes en dessous (valve-inblock engine, low set valve engine).

Un alliage spécial, offrant une bonne résistance à la fatigue et une grande tenue thermique, sert pour sa fabrication. Comme ce métal est différent de celui qu'on utilise pour réaliser la culasse ou le bloc-cylindres, on parle de siège rapporté (valve seat insert, built-in valve seat).

La tige de soupape coulisse à l'intérieur d'un élément fixe de la culasse ou du bloc-cylindres, le guide de soupape (valve guide), qui, lui aussi, est rapporté. En plus de guider la soupape, cette pièce, qui est disposée dans une chambre ou chemise d'eau (water jacket) du moteur, assure l'évacuation de la chaleur à partir de la tête. C'est là une fonction importante, surtout dans le cas des soupapes d'échappement (exhaust valve) que lèchent à intervalles réguliers les gaz brûlés très chauds produits par la combustion. Sur les moteurs poussés même, on améliore l'évacuation de la chaleur par la tige en y ménageant un chambrage. Ce dernier est rempli par exemple de sodium qui, en fondant vers $100^{\circ} \mathrm{C}$, absorbe de la chaleur. Ce sont les fameuses soupapes au sodium (sodium-cooled valve) équipant les moteurs de compétition (racing engine).

\section{La commande de soupape}

La pièce qui assure la synchronisation de l'ouverture des soupapes avec le mouvement des pistons, donc avec le mouvement du vilebrequin (crankshaft), est l'arbre à cames (camshaft) ou arbre de distribution (timing shaft, timer shaft). Elle porte, en nombre égal à celui des soupapes à commander, de petits disques comportant chacun une pointe orientée selon une direction précise. Ce sont les cames $(\mathrm{cam})$, dont les pointes, qualifiées de bossages (lobe), servent à transformer le mouvement circulaire du vilebrequin en un mouvement de va-et-vient rectiligne (to-and-fro motion, reciprocating motion) qui est communiqué aux soupapes par l'intermédiaire d'une succession de pièces mécaniques (mechanical part) formant avec la came ce qu'on appelle la commande de soupape (valve train).

Les dispositions des soupapes

La distribution par soupapes en dessous

Initialement, la distribution se faisait au moyen de soupapes latérales ou soupapes en chapelle (valve-on-the-side). On parlait de moteurs à soupapes en dessous (valve-in-block engine, side valve engine, flat head engine, low set valve engine). La levée (lift) des soupapes s'effectuait de bas en haut. Aucun basculeur 
ou culbuteur (rocker arm) n'était nécessaire pour renvoyer vers le bas le mouvement rectiligne ascendant obtenu grâce aux cames.

Deux variantes de la distribution par soupapes en dessous sont à distinguer : celle du moteur à soupapes latérales (L-head engine) où les deux types de soupapes sont d'un même côté du bloc-cylindres et celle du moteur à soupapes bilatérales (T-head engine) où les soupapes d'admission sont d'un côté et les soupapes d'échappement de l'autre.

La distribution par soupapes en dessous, si elle conduisait à une grande simplicité de réalisation, n'était pas sans inconvénient. Le fluide moteur (working fluid), qui décrit une trajectoire en double coude tant à l'admission qu'à l'échappement, se trouve entravé dans son mouvement et les pertes de charge (loss of head) sont importantes. Dans ces conditions le coefficient ou taux de remplissage (volumetric efficiency, charge efficiency, breathing ability) - que l'on définit comme le rapport entre le volume qu'occuperaient les gaz admis dans un cylindre à la température et à la pression atmosphérique, et le volume balayé par le piston durant une course (stroke), c'est-à-dire la cylindrée unitaire (piston displacement) - ne peut être que faible. Or la puissance développée (developed, cranked out, pumped out) par le moteur est directement liée à sa valeur.

Les constructeurs ont donc cherché à améliorer ce coefficient de remplissage. Une solution, d'abord retenue pour les moteurs équipant les voitures de sport ou de compétition, s'est imposée. Il s'agit de la distribution par soupapes en tête ou distribution par soupapes en dessus.

La distribution par soupapes en tête

Sur les moteurs à soupapes en tête (overhead valve engine, $O H V$ engine, valve-in-head engine, I-head engine), les soupapes sont disposées dans la culasse, la queue dirigée vers le haut, en sens opposé de l'embiellage. Grâce à cette disposition, l'admission et l'échappement des gaz sont directs. Le remplissage des cylindres se fait donc bien. Mais l'ouverture des soupapes appelle un mouvement de haut en bas pour ces dernières. Si l'arbre à cames se situe en dessous des queues de soupapes, dans le carter (crankcase) par exemple, il faut renvoyer vers le bas le mouvement rectiligne ascendant que produisent les cames.

La commande de soupape s'en trouve «alourdie», car elle doit comporter, en plus d'un poussoir (cam rider, cam follower, cam tappet, valve lifter) et d'une tige de poussoir (push rod, pushrod, tappet stem), une pièce capable d'assurer cette fonction de renvoi, le culbuteur ou basculeur. Le plus souvent, les culbuteurs, dont la partie centrale est perforée, s'enchâssent sur une tige creuse disposée au-dessus de la culasse et dénommée axe de culbuteurs (rocker arm shaft). Chaque culbuteur peut osciller autour de cet axe sous l'effet de l'impulsion que lui communique, à l'une de ses extrémités, la tige de poussoir. En basculant de cette façon, il fait se décoller de son siège la soupape dont la queue repose sur l'autre extrémité du culbuteur.

Avec les culbuteurs et les paliers servant à le supporter, l'axe des culbuteurs forme la rampe de culbuteurs (rocker arm assembly). Quand la distribution 
(timing gear) d'un moteur met en jeu un tel mécanisme, à la fois pour les soupapes d'admission et pour les soupapes d'échappement, on dit du moteur qu'il est culbuté. La culbuterie (rocker gear) désigne l'ensemble des culbuteurs et des pièces qui commandent leurs mouvements : c'est l'ensemble des commandes de soupape sur un moteur culbuté.

Un moyen terme : la distribution mixte

La distribution mixte représente une variante peu répandue de la distribution par soupapes en tête. Un moteur qui met en œuvre ce type de distribution est un moteur semi-culbuté. On dit encore un moteur à soupapes mixtes ( $F$-head engine). Pour chaque cylindre, l'une des soupapes est disposée dans la culasse et l'autre dans le bloc-cylindres. En principe c'est la soupape d'admission qui se trouve en tête, au-dessus du cylindre.

Un nouveau progrès : l'arbre à cames en tête

La distribution par soupapes en tête classique, à arbre à cames dans le carter, présente l'inconvénient de manquer de précision. Aux régimes élevés, l'inertie de la commande de soupape provoque l'affolement (floating, fluttering, bouncing) des soupapes qui ne sont pas rappelées assez vite par les ressorts (valve spring). Il n'y a plus obturation véritable de la lumière d'admission ou d'échappement du fait que la portée ne vient plus s'appuyer sur le siège de soupape, faute de temps. Sous cette forme, la distribution peut donc encore limiter les performances d'un moteur.

En vue de surmonter le problème, sans recourir pour le rappel des soupapes à un mécanisme d'entraînement forcé (positive drive) qui se substitue au ressort, les constructeurs en sont venus depuis quelques années à disposer l'arbre à cames au-dessus de la culasse, de façon notamment à supprimer les tiges de poussoir et à rendre ainsi la commande des soupapes plus directe.

Les moteurs pour lesquels cette disposition constructive est retenue sont des moteurs à arbre à cames en tête ou en dessus (overhead camshaft engine, $O H C$ engine, overhead cam engine). La commande des soupapes par arbre à cames en tête ne pouvant être qu'un attribut des moteurs à distribution par soupapes en tête, ce dernier aspect n'est pas rappelé dans le terme servant à désigner les moteurs qui l'utilisent : il est implicite.

Quand l'arbre de distribution ainsi placé en tête est unique, il s'agit d'un moteur à simple arbre à cames en tête (single overhead camshaft engine, SOHC engine). Si par contre les cames d'admission (intake cam) sont montées sur un arbre et les cames d'échappement (exhaust cam) sur un autre, on parle de moteur à double arbre à cames en tête ( $t$ win overhead camshaft engine, dual overhead camshaft engine, DOHC).

Un dernier raffinement : l'attaque directe des soupapes

Avec la distribution par arbre à cames en tête, simple ou double, les soupapes sont toujours placées au-dessus des cylindres mais elles ne sont pas nécessairement culbutées. La commande des soupapes peut ne pas se faire au moyen de culbuteurs. C'est alors la came qui attaque directement la queue de soupape. La distribution est du type à arbre à cames en tête et attaque directe (overhead camshaft direct type layout). 


\section{VOCABULAIRE FRANÇAIS-ANGLAIS}

Admission - intake. Première phase du cycle mécanique de fonctionnement d'un moteur à combustion intermittente, durant laquelle le fluide moteur gazeux (air ou air chargé de vapeurs d'essence) est aspiré dans le cylindre. Le mécanisme de distribution règle l'instant au cours du cycle auquel commence cette phase.

Affolement - floating. Anomalie de fonctionnement du mécanisme de distribution se produisant aux régimes élevés: défaut de fermeture des soupapes que les ressorts ne rappellent pas assez vite sur leur siège.

Allumeur - distributor. Boîtier à l'intérieur duquel se trouvent groupés deux éléments fonctionnels très importants d'un circuit d'allumage par batterie : le rupteur, qui s'insère dans le circuit primaire; et le distributeur, placé au-dessus, et appartenant, lui, au circuit secondaire. À ces éléments s'ajoutent souvent un dispositif d'avance centrifuge et un dispositif d'avance à dépression.

Arbre à cames - camshaft. Arbre sur lequel sont calés des excentriques, les cames, commandant la levée des soupapes. Le décalage angulaire entre les lobes des cames assure que les soupapes, dans les différents cylindres, s'ouvrent à intervalles réguliers selon un ordre établi, en synchronisme avec le mouvement des pistons.

Arlbre de distribution - timing shaft, timer shaft. Synonyme de arbre à cames.

Axe de culbuteur - rocker arm shaft. Dans un moteur culbuté ou semi-culbuté, axe monté sur le dessus de la culasse et sur lequel s'enchâssent les culbuteurs qui peuvent osciller autour de lui.

Basculeur. Synonyme de culbuteur.

Bloc-cylindres - cylinder block.

Bossage (de came) - (cam) lobe.

Came - cam. Petite pièce calée sur l'arbre à cames, constituant le premier élément de chaque cornmande de soupape. Elle transforme le mouvement de rotation du vilebrequin en un mouvement rectiligne alternatif de la tige de poussoir et de la soupape qui lui sont associées.

Came d'échappement - exhaust cam. Came de l'arbre de distribution commandant la levée d'une soupape d'échappement.

Came d'admission - intake cam. Came de l'arbre de distribution commandant la levée d'une soupape d'admission.

Capsulisme, moteur à. Synonyme de moteur volumétrique.

Carter - crankcase. (En français, carter s'emploie par abréviation de carter moteur.)

Chambre d'eau - water jacket. Nom donné aux cavités ménagées autour des cylindres et des évidements de la culasse les surmontant, dans la masse du bloc moteur. Ces cavités communiquent entre elles et forment un circuit où circule le fluide de refroidissement. On emploie aussi le terme chemise d'eau.

Coefficient de remplissage - volumetric efficiency.

Collerette - collar. Surface de raccordement entre la tête et la tige d'une soupape.

Collet de soupape - valve head face. Partie de la tête de soupape affectant une forme tronçonique, qui à la retombée de la soupape vient s'appuyer contre le siège de soupape èt réalise un joint hermétique avec ce dernier.

Commande de soupape - valve train. Mécanisme réglant l'ouverture et la fermeture d'une soupape, qui comprend, en plus d'une came calée sur l'arbre de distribution, un ensemble de pièces mécaniques servant de relais entre cette dernière et la soupape commandée. Dans le cas d'un moteur culbuté, cet ensemble est formé d'un poussoir, d'une tige de poussoir, d'un culbuteur et d'un ressort pour le rappel de la soupape sur son siège. Le terme anglais valve train correspond à l'ensemble constitué par la commande de soupape et la soupape.

Course - stroke. Distance parcourue par un piston dans le cylindre, entre le point mort haut et le point mort bas.

Culasse - cylinder head, head. Dans un moteur à pistons, pièce surmontant le bloc-cylindres. Elle comporte des évidements qui ferment les cylindres et forment l'espace mort ou chambre de combustion lorsque le piston atteint le point mort haut, c'est-à-dire le sommet de sa course pour un noteur vertical. Des canaux y sont ménagés pour permettre, d'une part, la circulation d'eau de refroidissement et, d'autre part, l'admission de la charge ainsi que l'évacuation des gaz brûlés après la combustion.

Autrefois, cette pièce était venue de fonderie avec le bloc-cylindres, et l'on parlait de moteur à cylindres borgnes. Désormais, il s'agit d'une pièce rapportée sur le bloc-cylindres, se présentant le plus souvent sous la forme d'un moulage en aluminium. 
Culbuterie - rocker arm. Ensemble des culbuteurs et des pièces commandant leur mouvement, sur un moteur culbuté.

Culbuteur - rocker arm, rocker lever. Sur un moteur culbuté, à arbre à cames dans le carter, levier articulé servant à renvoyer le mouvement de commande des soupapes par dessus la culasse. Les commandes de soupape comportent également des culbuteurs sur les moteurs à arbre à cames en tête, mais seulement lorsque l'attaque des soupapes n'est pas directe.

Cylindrée unitaire - piston displacement. Pour un moteur à combustion interne de type volumétrique, différence entre le volume maximal de la chambre de combustion et le volume minimal. Cette définition s'applique au moteur à pistons de type classique, mais peut s'étendre au moteur rotatif. Par abréviation, on emploie le terme cylindrée seul.

Distributeur - distributor. Partie de l'allumeur servant à répartir, entre les différents cylindres d'un moteur à explosion, les impulsions de haute tension produites par la bobine d'allumage en conjonction avec les différents éléments du circuit primaire.

Distribution - timing. Fonction mise en ouvre sur les moteurs à combustion intermittente (à explosion ou diesel). Elle consiste à mettre périodiquement en communication les cylindres avec les conduits d'admission et d'échappement. La plupart des moteurs à quatre temps utilisent la distribution par soupapes.

Distribution sans soupape - valveless timing.

Échappement - exhaust. Dernière phase du cycle mécanique de fonctionnement d'un moteur à combustion intermittente, durant laquelle les produits de la combustion sont évacués dans l'atmosphère. Le mécanisme de distribution règle l'instant au cours du cycle auquel commence cette phase. Fluide moteur - working fluid. Dans un moteur thermique, fluide recevant de l'énergie sous forme de chaleur d'une source chaude et la restituant en partie sous forme de travail à un organe moteur (piston ou roue de turbine) et en partie sous forme d'énergie thermique résiduelle à une source froide. Levée - lift. Mouvement exécuté par une soupape d'admission ou d'échappement, suivant la direction de son axe longitudinal, lorsqu'elle décolle de son siège.

Lumière d'admission - intake port. Ouverture de la chambre de combustion, pratiquée dans la culasse ou le bloc-cylindres et où débouche un conduit d'admission communiquant avec l'atmosphère par l'intermédiaire de la tubulure d'admission (et du carburateur s'il y en a un).

Lumière d'échappement - exhaust port. Ouverture de la chambre de combustion, pratiquée dans la culasse ou le bloc-cylindres et d'où part un conduit d'échappement communiquant avec l'atmosphère par l'intermédiaire du collecteur d'échappement.

Mécanisme d'entraînement forcé - positive drive.

Moteur à arbre à cames en tête - overhead camshaft engine, $O H C$ engine. Moteur à soupapes en tête dont l'arbre à cames se trouve placé au-dessus de la culasse et non dans le carter. Une telle disposition rend la commande des soupapes plus franche.

Moteur à capsulisme. Synonyme de moteur volumétrique.

Moteur à distribution mixte. Synonyme de moteur à soupapes mixtes.

Moteur à double arbre à cames en tête - dual overhead camshaft engine, DOHC engine.

Moteur à simple arbre à cames en tête - single overhead camshaft engine, SOHC engine.

Moteur à distribution mixte $-F$-head valve engine. Moteur dont la distribution utilise une soupape latérale et une soupape en tête, cette dernière étant réservée le plus souvent à l'admission. Cette disposition permet de donner à la soupape d'admission, placée en dessus, une très forte section qui garantit une excellente alimentation en gaz du moteur, sans augmenter exagérément la surface de la chambre de combustion.

Moteur à soupapes bilatérales - T-head engine. Moteur à soupapes en dessous dont les soupapes sont disposées de part et d'autre des cylindres.

Moteur à soupapes en dessous - valve in-block engine. Moteur dont la distribution est assurée par des soupapes disposées d'un côté des cylindres et que commande un arbre à cames placé dans le carter. L'ouverture des soupapes se fait de bas en haut.

Moteur à soupapes en tête - overhead valve engine, $O H V$ engine. Moteur dont la distribution est assurêe par des soupapes disposées dans la culasse et ayant leur queue dirigée vers le haut. L'ouverture des soupapes est obtenue en donnant à ces dernières un mouvement de haut en bas transmis à partir d'une came de l'arbre de distribution.

Moteur à soupapes latérales - L-head engine, side valve engine. Moteur à soupapes en dessous dont les soupapes sont toutes disposêes d'un mêrne côté du bloc-cylindres.

Moteur à soupapes mixtes. Synonyme de moteur à distribution mixte. 
Mloteur de type turbomachine - non-displacement type engine. Machine prélevant de l'énergie à un flux continu de fluide, qu'elle transforme en énergie mécanique utilisée pour maintenir en mouvement une autre machine.

Moteur semi-culbuté. Synonyme de moteur à distribution mixte.

Moteur thermique - heat engine. Machine produisant du travail (énergie motrice) à partir de chaleur. La conversion utilise un fluide moteur qui reçoit de la chaleur d'une source chaude grâce à un échangeur thermique ou plus directement en participant à une combustion. Une partie seulement de la chaleur reçue est transformée en travail quand le fluide moteur se détend dans une capacité contenant un organe mécanique récepteur (piston ou roue de turbine). La chaleur résiduelle est restituée à une source froide, que peut représenter l'environnement, l'atmosphère. Moteur volumétrique - positive displacement engine, displacement-type engine. Moteur thermique utilisant l'énergie potentielle d'un gaz (le fluide moteur) que l'on fait se détendre, pour produire du travail, à l'intérieur d'une enceinte fermée dont le volume est variable. Ce gaz peut avoir reçu de la chaleur préalablement dans un organe distinct : c'est le cas des moteurs à combustion externe, dont le type est la machine à vapeur. Mais l'apport de chaleur peut aussi avoir lieu à l'intérieur de l'organe où se produit la détente: c'est le cas des moteurs à combustion interne. Le moteur à explosion, le diesel et le moteur rotatif Wankel illustrent cette deuxième grande classe de moteurs volumétriques. Aux moteurs volumétriques s'opposent les machines cinétiques ou turbomachines. Pertes de charge - loss of head. Diminution de l'énergie interne d'un fluide en écoulement, par suite des frottements contre les parois ou de l'existence de singularités le long de la conduite.

Portée de soupape. Synonyme de collet de soupape.

Poussoir - cam follower, valve lifter. Élément de la commande de soupape dont l'une des extrémités, en appui permanent sur une came de l'arbre de distribution, suit le contour de celle-ci. De forme cylindrique, il a pour fonction de guider la pièce mécanique de la commande juste en aval, qui peut être une tige de poussoir ou directement la soupape.

Queue (de soupape) - (valve) tip, (valve) stem end. Extrémité de la tige de soupape qui reçoit la coupelle d'appui du ressort de soupape. Suivant le type de distribution, elle est attaquée par l'extrémité d'une tige de poussoir, un culbuteur, un poussoir, ou directement par une came.

Rampe de culbuteurs - rocker gear assembly. Dans un moteur culbuté, ensemble formé par les culbuteurs, l'axe de culbuteurs sur lesquels ces derniers sont enchâssés et les supports de cet axe. Sìge de soupape - valve seat. Pièce le plus souvent rapportée dans la lumière d'admission ou d'échappement, sur la surface de laquelle la portée de la soupape, qui affecte la même forme généralement tronçonique, vient s'appuyer à la fin de l'admission ou de l'échappement de façon à fermer la chambre de combustion.

Siège de soupape rapporté - valve seat insert.

Soupape - valve. Obturateur à levée que l'on utilise sur la plupart des moteurs à combustion intermittente pour mettre en communication les chambres de combustion avec l'atmosphère pendant certains temps du cycle.

Soupape au sodium - sodium-cooled valve.

Soupape plate - flat valve, plate valve.

Taux de remplissage. Synonyme de coefficient de remplissage.

Tête de soupape - valve head. Partie d'une soupape opposée à la queue et remplissant à proprement parler la fonction d'obturation.

Tige de soupape - valve stem. Partie cylindrique et allongée d'une soupape, se terminant par la queue. En plus d'assurer une fonction de guide, elle sert à évacuer la chaleur.

Vilebrequin - crankshaft.

\section{LEXIQUE ANGLAIS-FRANÇAIS}

Bouncing, (valve). Syn. de valve floating.

Breathing ability. Syn. de volumetric efficiency.

Cam - came.

Cam follower - poussoir.

Cam lobe - bossage de came.

Cam rider. Syn. de cam follower.

Camshaft - arbre à cames.

Cam tappet. Syn. de cam follower.

Charge efficiency. Syn. de volumetric efficiency. 
Crankcase - carter.

Crankshaft - vilebrequin.

Cylinder block - bloc-cylindres.

Cylinder head - culasse.

Disc, valve - tête de soupape.

Displacement, piston - cylindrée unitaire.

Displacement-type engine - moteur volumétrique.

Distributor - allumeur; distributeur (partie de l'allumeur).

DOHC engine. Abréviation de dual overhead camshaft engine.

Drive, positive - mécanisme d'entraînement forcé.

Dual overhead camshaft engine - moteur à double arbre à cames en tête.

Efficiency, charge. Syn. de volumetric efficiency.

Efficiency, volumetric - coefficient de remplissage.

Exhaust - échappement.

Exhaust cam - came d'échappement.

Exhaust port - lumière d'échappement.

Exhaust valve - soupape d'échappement.

F-head engine - moteur à soupapes mixtes.

Face, valve head - portée (de soupape), collet.

Flat head engine - moteur à soupapes en dessous.

Flat valve - soupape plate, soupape à siège plan.

Floating, (valve) - affolement (des soupapes).

Follower, cam - poussoir.

Fluttering, (valve). Syn. de valve floating.

Head, (cylinder) - culasse.

Head, loss of - pertes de charge.

Head, valve - tête de soupape.

Heat engine - moteur thermique.

I-head engine. Syn. de overhead valve engine.

Insert, valve seat - siège (de soupape) rapporté.

Intake cam - came d'admission.

Jacket, water - chemise d'eau, chambre d'eau.

L-head engine - moteur à soupapes latérales.

Lift - levée.

Lifter, valve. Syn. de cam follower.

Lobe, (cam) - bossage (de came).

Loss of head - pertes de charge.

Low set valve engine - Syn. de valve-in-block engine.

Non-displacement type engine - moteur de type turbomachine.

OHC engine. Abréviation de overhead camshaft engine.

OHV engine. Abréviation de overhead value engine.

Overhead cam engine. Syn. de overhead camshaft engine.

Overhead camshaft direct type layout - distribution à arbre à cames en tête et commande directe.

Overhead camshaft engine - moteur à arbre à cames en tête.

Piston displacement - cylindrée unitaire.

Plate valve - soupape plate, soupape à siège plan.

Plug, valve - tête de soupape.

Port, exhaust - lumière d'échappement.

Port, intake - lumière d'admission.

Positive drive - mécanisme d'entraînement forcé.

Rider, cam. Syn. de cam follower.

Rocker arm - culbuteur, basculeur.

Rocker arm assembly - rampe de culbuteurs.

Rocker arm shaft - axe de culbuteurs.

Rocker gear - culbuterie.

Seat, valve - siège de soupape.

Side valve - soupape latérale, soupape en chapelle. 
Side valve engine. Syn. de L-head engine.

Single overhead camshaft engine - moteur à simple arbre à cames en tête.

Sodium-cooled valve - soupape au sodium.

SOHC engine. Abréviation de single overhead camshaft engine.

Stem end, valve - queue de soupape.

Stem, valve - tige de soupape.

Stroke - course.

T-Head engine - moteur à soupapes bilatérales.

Tappet, cam. Syn. de cam follower.

Timer shaft. Syn. de timing shaft.

Timing - distribution.

Timing shaft - arbre de distribution.

Timing gear - mécanisme de distribution, distribution.

Timing, valveless - distribution sans soupape.

Tip, valve - queue de soupape.

Twin overhead camshaft engine - Syn. de dual overhead camshaft engine.

Valve - soupape.

Valve disc - tête de soupape.

Valve floating - affolement des soupapes.

Valve head - tête de soupape.

Valve head face - portée (de soupape), collet.

Valve-in-block engine - moteur à soupapes en dessous.

Valve-in-head engine. Syn. de overhead valve engine.

Valve lifter. Syn. de cam follower.

Valve plug - tête de soupape.

Valve seat - siège de soupape.

Valve seat insert - siège (de soupape) rapporté.

Valve stem - tige de soupape.

Vallve stem end - Syn. de valve tip.

Valve tip - queue de soupape.

Valve train - commande de soupape.

Valveless timing - distribution sans soupape.

Volumetric efficiency - coefficient de remplissage.

Water jacket - chemise d'eau, chambre d'eau.

Working fluid - fluide moteur. 


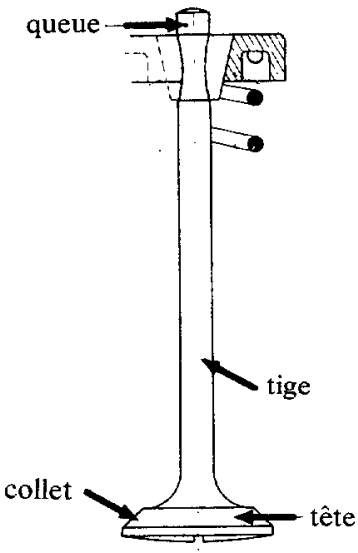

Soupape

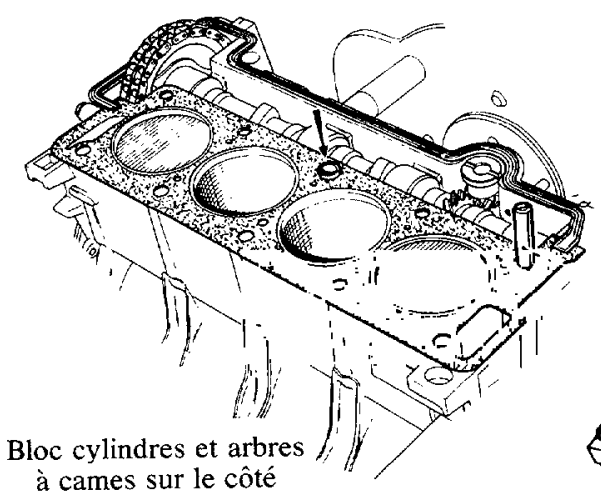
à cames sur le côté

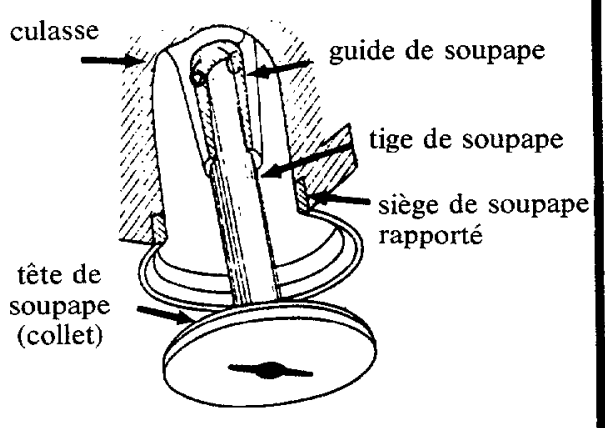

Soupape dans son guide et siège de soupape

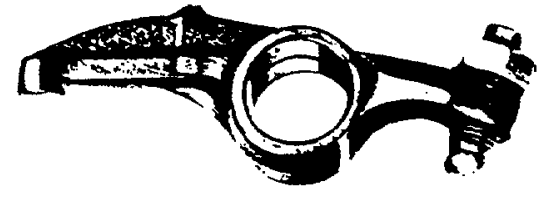

Culbuteur

axe de culbuteurs
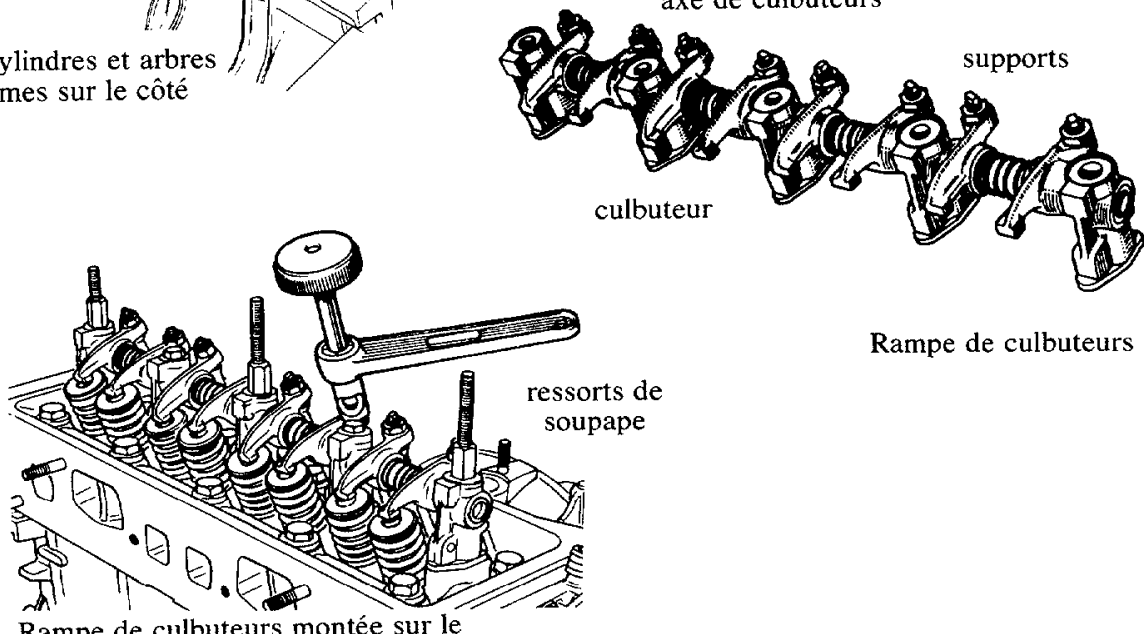

Rampe de culbuteurs

Rampe de culbuteurs montée sur

dessus de la culasse d'un moteur à quatre cylindres 

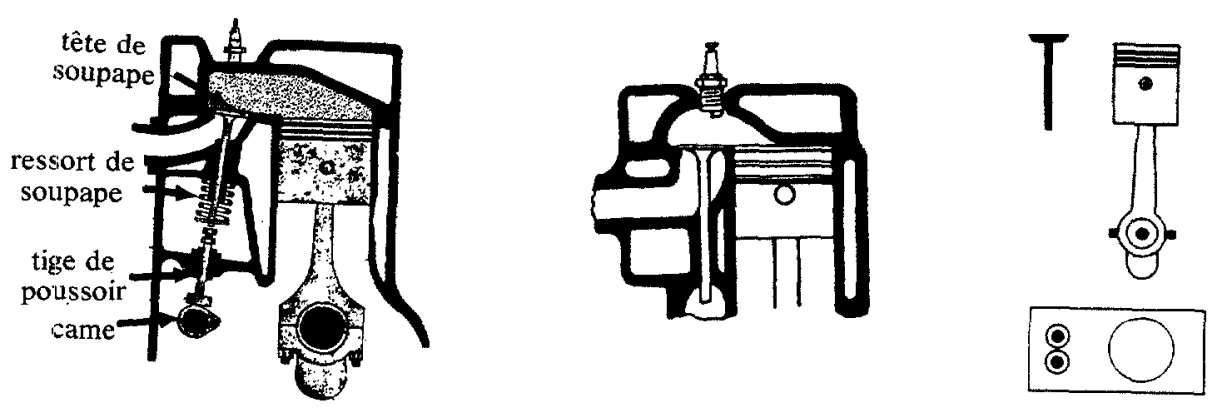

Moteur à soupapes latérales

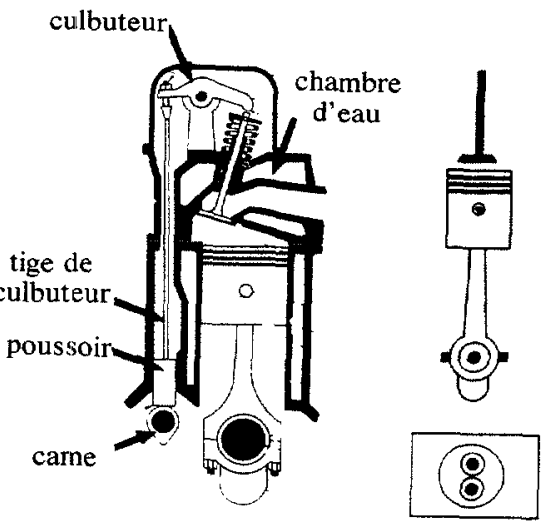

Moteur à soupapes en tête et arbre à cames dans le carter
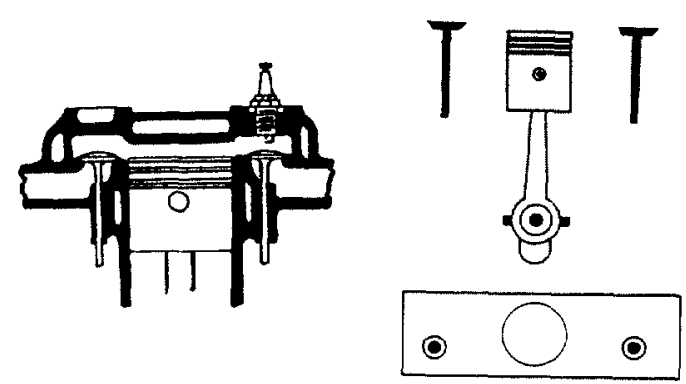

Moteur à soupapes bilatérales

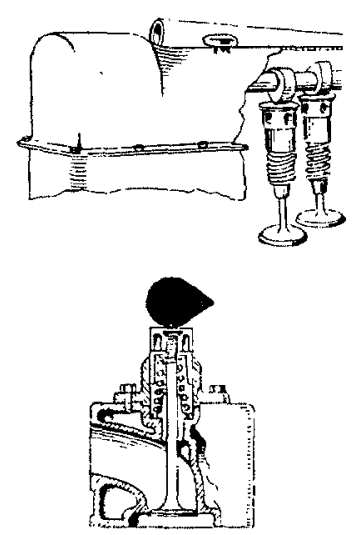

Moteur à simple arbre à cames en tête et attaque directe des soupapes 


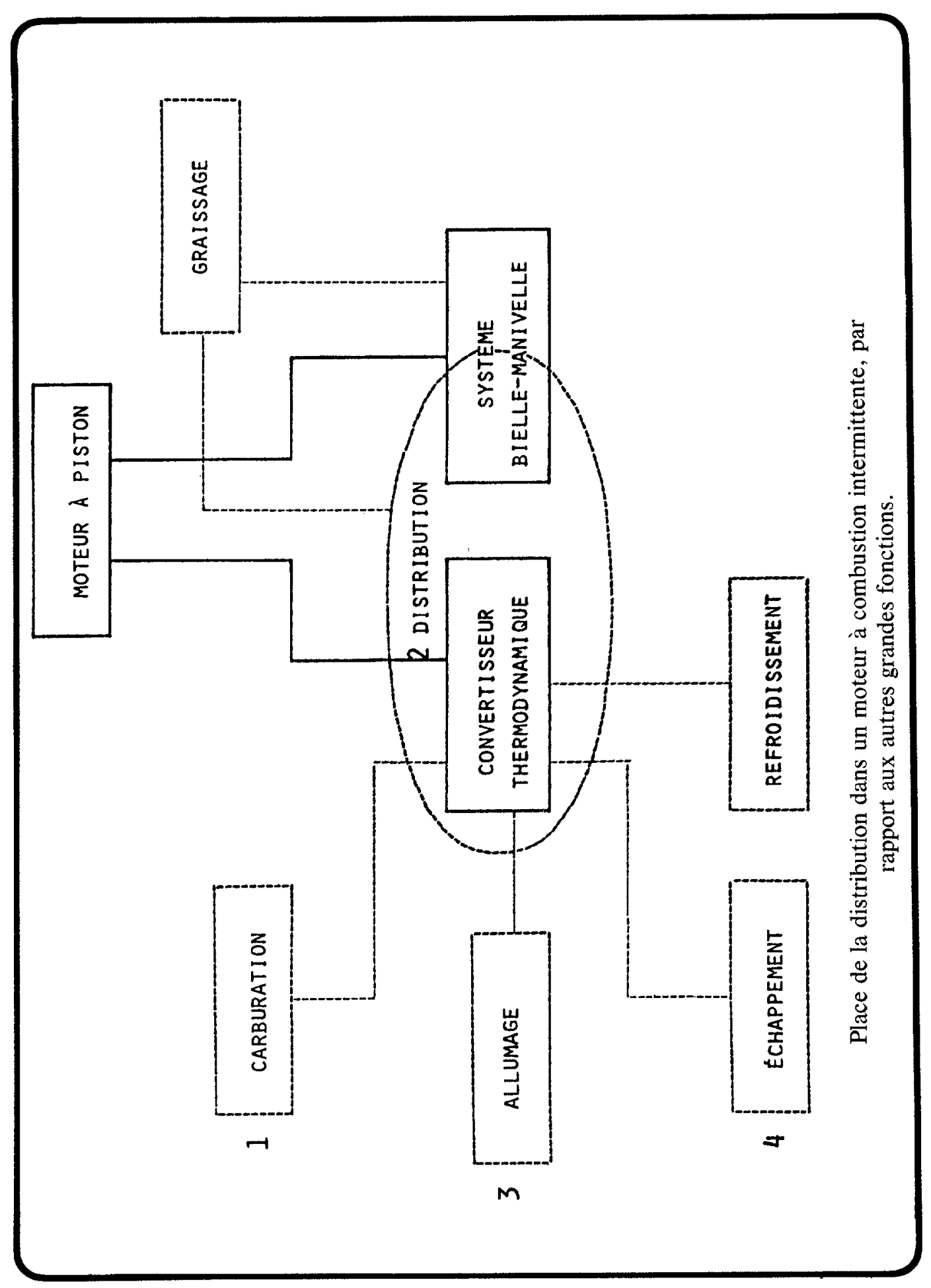




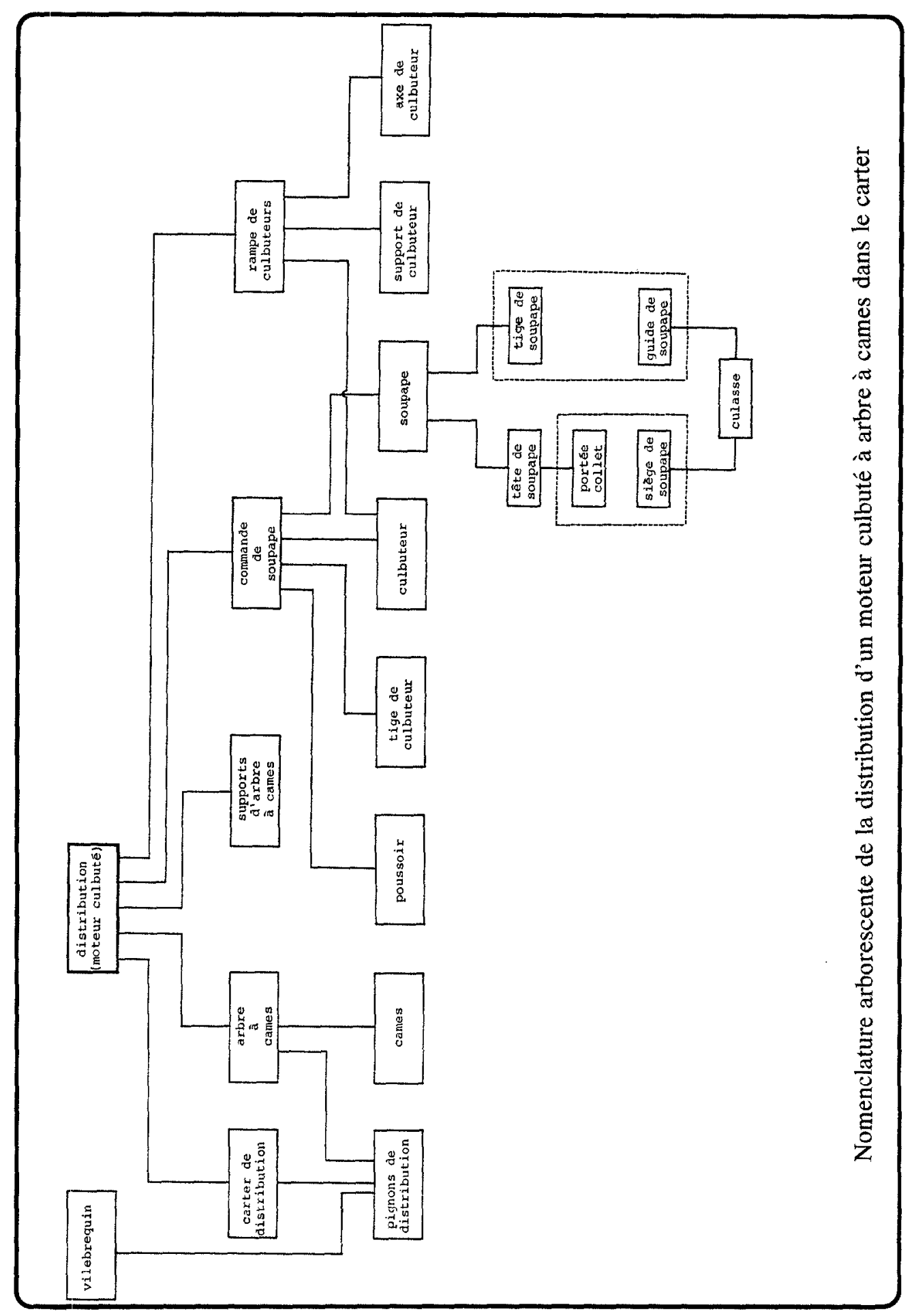


META, XXVII, 3

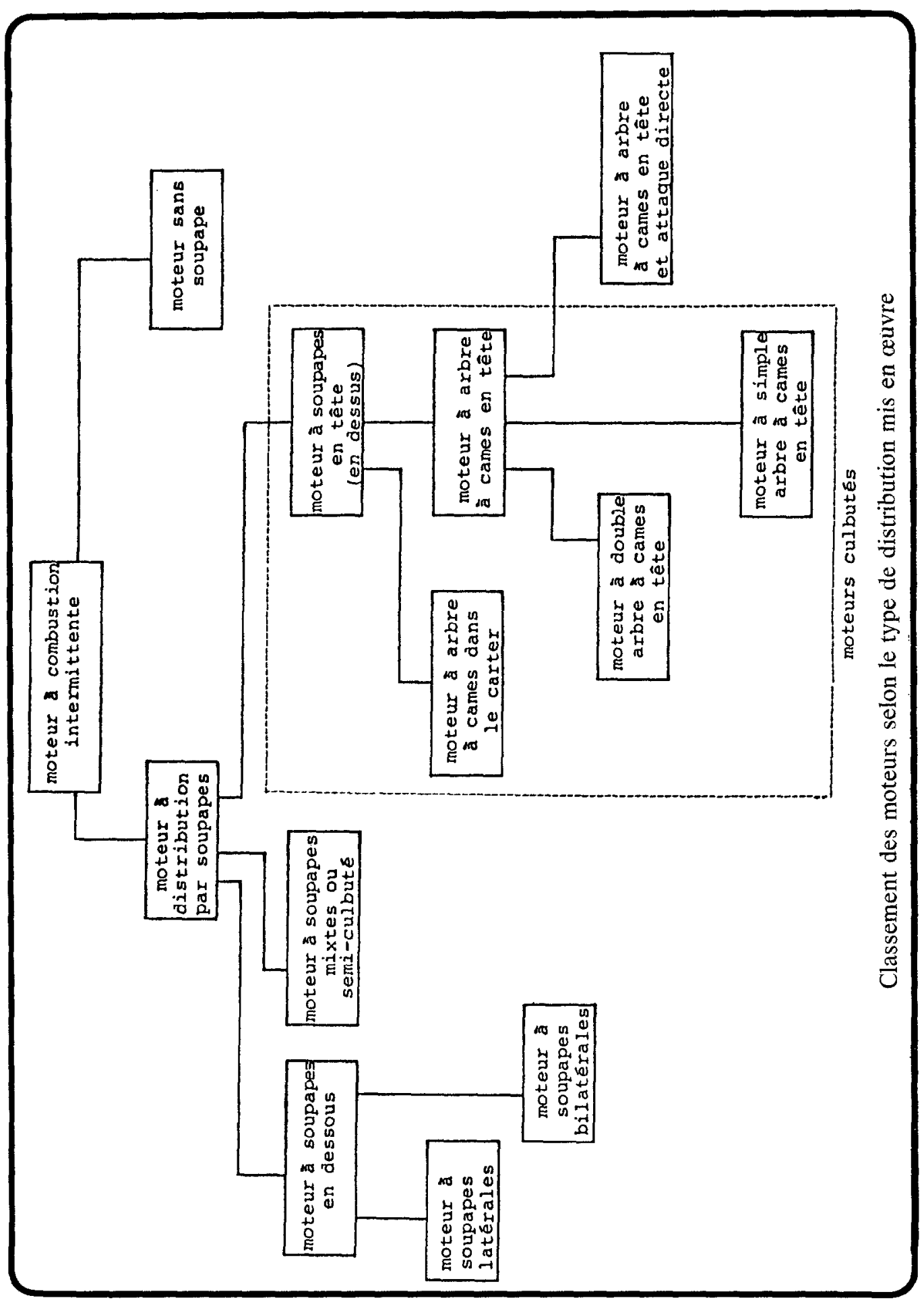

